\section{Biliary Excretion of Glutathione and Glutathione Disulfide in the Rat Regulation and Response to Oxidative Stress}

Bemhard H. Lauterburg, Charles V. Smith, Helen Hughes, and Jerry R. Mitchell

Department of Internal Medicine and Institute for Lipid Research, Baylor College of Medicine, Houston, Texas 77030
4 of reduced glutathione (GSH) and glutathione disulfide (GSSG) and responses to selected model toxins were examined in male Sprague-Dawley rats. In control and phenobarbital-pretreated rats in which the intrahepatic concentration of GSH was modulated by the administration of diethyl maleate or acetaminophen, the biliary concentration of GSH was consistently lower than, but directly proportional to, the intrahepatic concentration of GSH. Furthermore, increments in bile flow produced by the infusion of sulfobromophthalein (BSP)-glutathione were associated with proportional increases in the biliary excretion of GSH, suggesting that GSH passes into bile passively along a concentration gradient. In contrast, GSSG appears to be secreted into bile against a steep concentration gradient. An increased hepatic production and biliary excretion of GSSG resulted from the administration of $t$-butyl hydroperoxide. Measurement of biliary GSSG and BSP during a constant infusion of the GSH adduct of BSP indicated that GSSG shares a common excretory mechanism with GSH adducts. Diquat, nitrofurantoin, and paraquat also markedly stimulated the biliary excretion of GSSG. On a molar basis, these compounds generated much more GSSG than a direct substrate for glutathione peroxidase such as $t$-butyl hydroperoxide, indicating that the compounds undergo redox-cycling with concomitant production of hydrogen peroxide. Aminopyrine $(0.8 \mathrm{mmol} / \mathrm{kg})$ also significantly increased biliary GSSG. This increase, however, was associated with a proportional increase in bile flow and in the biliary excretion of GSH such that the GSSG/GSH

Received for publication 21 December 1982 and in revised form 2 August 1983.

J. Clin. Invest.

(c) The American Society for Clinical Investigation, Inc.

0021-9738/84/01/0124/10 \$1.00

Volume 73, January 1984, 124-133 ratio in bile did not change. Acetaminophen and chloroform, two compounds generating electrophilic metabolites that deplete intrahepatic GSH, led to a progressive decrease in the biliary excretion of GSH and GSSG. Furosemide and dimethylnitrosamine, the electrophilic metabolites of which do not deplete hepatic GSH, minimally altered biliary GSH and GSSG. Similarly, carbon tetrachloride and iproniazid, which yield organic radical metabolites that can peroxidize membrane lipids, did not increase the biliary excretion of GSSG. This finding indicates that membrane-bound lipid hydroperoxides may not be good substrates for glutathione peroxidases. The measurement of the biliary excretion of GSSG and of the GSSG/GSH ratio in bile is a sensitive index of oxidative stress in vivo and thus complements other in vivo parameters for the study of reactive intermediates of xenobiotics such as the determination of covalent binding, the formation of lipid hydroxy acids, and the depletion of intracellular GSH.

\section{Introduction}

It recently has been recognized that many drugs and xenobiotics are metabolically activated to reactive intermediates that may cause cell injury in the liver, kidney, and lung (1-3). A valuable tool for demonstrating the formation of reactive intermediates in vivo has been the modulation of tissue injury by inhibition or induction of the drug-metabolizing enzymes while simultaneously measuring the extent of covalent binding of part of the parent compound to tissue macromolecules. However, a number of compounds may exert their toxicity by the generation of reactive oxygen species rather than by the formation of a reactive intermediate of the parent molecule (4). In contrast to compounds generating electrophilic intermediates, such as acetaminophen, bromobenzene, and many furans and thiophenes, or those forming alkylating radicals, such as iproniazid, halothane, and carbon tetrachloride, the generation of reactive oxygen intermediates is not associated with the covalent binding of part of the original molecule to lipids or proteins, and therefore covalent binding cannot be used as an index for the formation 
of a reactive intermediate (5). Thus, evidence for the involvement of reactive oxygen species in toxic cell damage in vivo is circumstantial. Modulation of toxin-induced injury by variation of oxygen tension in the environment $(6-8)$ is usually considered as evidence for the involvement of oxygen intermediates in the toxic process in vivo $(3,4)$. Similarly, an increase in toxininduced injury in animals deficient in either vitamin $\mathrm{E}$ or selenium-dependent glutathione peroxidase suggests the involvement of reactive oxygen species because these deficiency states impair the cellular defense against oxygen toxicity.

Accordingly, for the elucidation of mechanisms of toxicity of compounds that are metabolically activated but do not covalently bind to tissue macromolecules, a more direct index of oxidative stress is necessary. The glutathione peroxidases are extremely important in protecting the cell from oxidative stress by reducing hydrogen peroxide $\left(\mathrm{H}_{2} \mathrm{O}_{2}\right)$, thereby stoichiometrically oxidizing glutathione (GSH) to glutathione disulfide (GSSG) in the process $(9,10)$. Although much of the generated GSSG will be converted intracellularly back to GSH by glutathione reductase, a small amount, but proportional to the GSSG concentration, will be excreted in bile (11). Whereas most of the hepatic production of GSH in the absence of an oxidative stress is excreted into plasma $(12,13)$, GSSG is preferentially excreted into bile (14) such that biliary GSSG should be a sensitive index of oxidant stress in the liver.

In addition to the rate of intracellular formation of GSSG, however, other factors such as the intracellular concentration of GSH, the biliary concentration of GSH and rate of autoxidation to GSSG, the rate of bile flow and the concomitant biliary excretion of other reaction products of GSH may influence the rate of excretion of GSSG and the GSSG/GSH ratio in bile. Thus, a better understanding of the regulation of the biliary excretion of GSSG and GSH is a prerequisite for the evaluation of the biliary excretion of GSSG as an index of oxidative stress. In the following studies we have characterized the determinants of the biliary excretion of GSH and GSSG in the rat. We then examined the response of biliary GSH and GSSG to the administration of selected model toxins thought to exert their toxicity either through the generation of reactive oxygen species or through electrophilic metabolites or alkylating radical intermediates $(5)$.

\section{Methods}

The biliary excretion of GSH and GSSG was measured in male SpragueDawley rats (Timco Breeding Laboratories, Houston, TX) weighing 200-250 g. The animals had free access to food and water up to the morning of the experiment. After the injection of $50 \mathrm{mg} / \mathrm{kg}$ i.p. of pentobarbital, the common bile duct was cannulated with PE-10 polyethylene tubing. The animals' temperature was measured rectally and kept between $37^{\circ}$ and $38^{\circ} \mathrm{C}$ with a heating lamp. Bile was collected for periods of $10-15 \mathrm{~min}$ in preweighed tubes containing $0.1 \mathrm{ml}$ of $4 \%$ sulfosalicylic acid to minimize extrahepatic oxidation of GSH (15)

To establish the relationship between intrahepatic and biliary concentration of GSH, diethyl maleate, $1 \mathrm{ml} / \mathrm{kg}$ i.p. in corn oil, or acetaminophen, $1 \mathrm{~g} / \mathrm{kg}$ i.p. in saline, was administered to control and phe- nobarbital-pretreated rats $(80 \mathrm{mg} / \mathrm{kg}$ i.p. daily for $4 \mathrm{~d})$ to deplete intrahepatic GSH. $1 \mathrm{~h}$ later, three bile samples were collected for the determination of GSH and a portion of the liver was removed for the measurement of GSH.

To test the hypothesis that GSSG and the GSH adduct of sulfobromophthalein (BSP-GSH) ${ }^{1}$ share a common excretory mechanism, BSP-GSH in saline was infused into a jugular vein of rats at a rate exceeding the maximal biliary excretory rate. The BSP-GSH adduct was synthesized according to Whelan et al. (16). Chromatography of the product on Avicel thin-layer chromatography plates using $n$-propanol/ water/acetic acid, 10:5:1, as the mobile phase showed that free BSP was not detectable and $>95 \%$ was present as BSP-GSH or diglutathionyl BSP (16). The compound was infused initially at a rate of $1,600 \mathrm{nmol} /$ $\min$ per $100 \mathrm{~g}$ for $5 \mathrm{~min}$ and then at a rate of $540 \mathrm{nmol} / \mathrm{min}$ per 100 g. Starting $30 \mathrm{~min}$ after onset of the infusion, bile was collected at 10min intervals. $30 \mathrm{~min}$ later, $t$-butyl hydroperoxide, $1.4 \mathrm{mmol} / \mathrm{kg}$ i.p., was administered in $0.9 \%$ saline. In similarly prepared animals, samples of liver tissue were obtained before and $10 \mathrm{~min}$ after the administration of $t$-butyl hydroperoxide for determination of the hepatic BSP-GSH content. To test the specificity of the observed competition between GSSG and BSP-GSH for biliary excretion, the experiments with $t$-butylhydroperoxide were repeated during an infusion of indocyanine green. Indocyanine green is not conjugated with GSH and has a much lower biliary transport maximum $(21 \mathrm{nmol} / \mathrm{min}$ per $100 \mathrm{~g}$ body weight [17]) than BSP-GSH. Therefore, the dye was infused at a rate of $25 \mathrm{nmol} /$ min per $100 \mathrm{~g}$ body weight in $0.9 \%$ saline containing $0.25 \%$ bovine serum albumin.

To evaluate the response of biliary GSH to oxidative stress, the biliary excretion of GSH and GSSG was measured before and after the intraperitoneal administration of the following compounds: $t$-butyl hydroperoxide, $0.28-14 \mathrm{mmol} / \mathrm{kg}$; diquat, $0.09 \mathrm{mmol} / \mathrm{kg}$; paraquat, 0.3 $\mathrm{nmol} / \mathrm{kg}$; nitrofurantoin, $0.2 \mathrm{mmol} / \mathrm{kg}$; aminopyrine, 0.08 and $0.8 \mathrm{mmol} /$ $\mathrm{kg}$; ethylmorphine, $0.08 \mathrm{mmol} / \mathrm{kg}$; acetaminophen, $6.6 \mathrm{mmol} / \mathrm{kg}$; chloroform, $12 \mathrm{mmol} / \mathrm{kg}$; furosemide, $1.2 \mathrm{mmol} / \mathrm{kg}$; dimethylnitrosamine, $0.4 \mathrm{mmol} / \mathrm{kg}$; carbon tetrachloride, $10 \mathrm{mmol} / \mathrm{kg}$; and iproniazid, 0.4 $\mathrm{mmol} / \mathrm{kg}$. All compounds were dissolved in $0.9 \%$ saline except for nitrofurantoin and the highest dose of $t$-butyl hydroperoxide, which were dissolved in dimethyl sulfoxide, and carbon tetrachloride and chloroform which were administered in mineral oil. The vehicles did not affect biliary GSH and GSSG. The choice of dosages was based on previous experiences with the particular compound and its toxic effects in the chosen animal model.

Analytical methods. After centrifugation and 10-fold dilution of the bile samples with $0.1 \mathrm{M}$ phosphate buffer, $\mathrm{pH} 7.4$, containing 2.5 $\mathrm{mM} \mathrm{Na}{ }_{2}$ EDTA, "total" GSH, i.e., GSH plus GSSG, was measured by the method of Tietze (18). The GSSG was determined after derivatization of GSH with 2-vinylpyridine (19). Recovery of GSH and GSSG added to bile collected in sulfosalicylic acid was $99 \pm 8$ and $96 \pm 10 \%$ $(n=8)$, respectively. The addition of $t$-butyl hydroperoxide, paraquat, or nitrofurantoin to native, nonacidified bile at a final concentration of $1 \mathrm{mM}$ did not accelerate the oxidation of GSH over $30 \mathrm{~min}$ at room temperature.

The GSH in $10 \%$ homogenates of hepatic tissue was measured by the method of Tietze (18) after precipitation of proteins with an equal volume of $4 \%$ sulfosalicylic acid.

Thin-layer chromatography of bile samples with the solvent system described above showed that all BSP was present as BSP conjugates.

1. Abbreviations used in this paper: BSP-GSH, GSH adduct of sulfobromophthalein. 


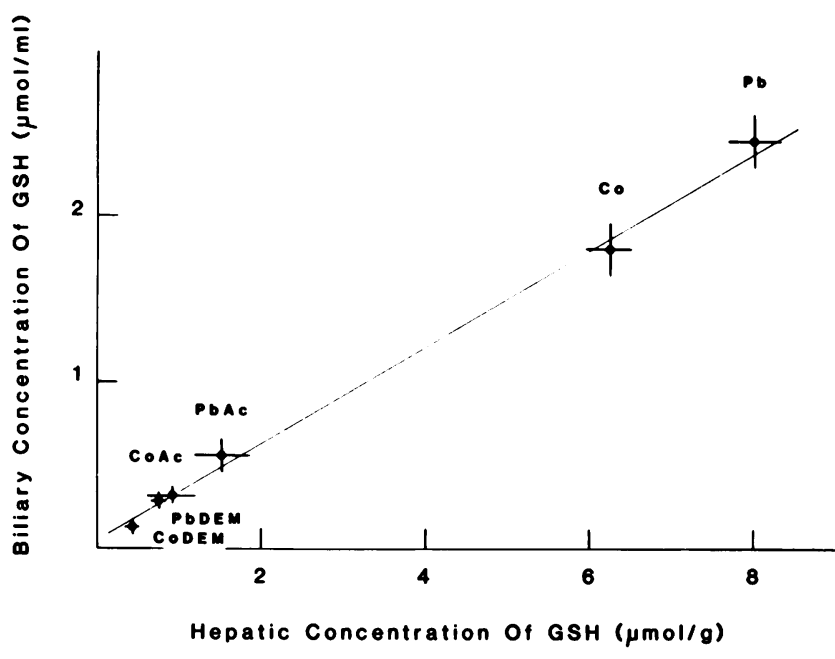

Figure 1. Biliary concentration of GSH as a function of the intrahepatic concentration of GSH. The average concentration of GSH in three bile samples obtained in each animal before death and the concentration of GSH in hepatic tissue were measured. Some animals were pretreated with phenobarbital, $80 \mathrm{mg} / \mathrm{kg}$ i.p., (Pb) for 4 d. Some of the control ( $\mathrm{Co})$ and phenobarbital-treated animals received diethyl maleate, $1 \mathrm{ml} / \mathrm{kg}$ (CoDEM, PbDEM), or acetaminophen, $1 \mathrm{~g} / \mathrm{kg}(\mathrm{CoAc}, \mathrm{PbAc})$, to further vary the intrahepatic concentration of GSH. Separate regression analyses for control and phenobarbital-treated animals did not reveal any significant differences between the two groups. Correlation coefficient: 0.99 , slope: 0.29 , mean $\pm \mathrm{SE}$ of groups of 6-12 animals.

For the determination of BSP conjugates in bile, samples were diluted 1,000 -fold with $0.1 \mathrm{~N} \mathrm{KOH}$, and absorbance was measured at $575 \mathrm{~nm}$ (16). Standard curves were obtained from bile samples to which known quantities of BSP were added. The BSP in 10\% homogenates of hepatic tissue was measured after precipitation of proteins with $0.04 \mathrm{vol}$ of $50 \%$ sulfosalicylic acid and dilution with $0.1 \mathrm{~N} \mathrm{KOH}$. For the measurement of indocyanine green, bile samples were diluted 100 -fold with $0.3 \%$ saline containing $0.25 \%$ bovine serum albumin and the absorbance was determined at $800 \mathrm{~nm}$ (17). Glutathione reductase in liver homogenates was measured according to Pinto and Bartley (20).

Calculations and statistics. For the calculation of GSH excretion, bile flow was determined from the weight of the excreted bile assuming a specific gravity of one. The increment in the biliary excretion of GSSG produced by any given compound was estimated by calculating the area under the GSSG excretion-time curve by the trapezoidal method and subtracting the basal excretion of GSSG.

All data are shown as mean \pm SE. Drug-induced changes in the biliary excretion of GSH and GSSG were assessed by the $t$ test for paired samples. For statistical treatment the two or three samples representing the basal excretion were pooled.

\section{Results}

The basal biliary excretion of GSH and GSSG (expressed as GSH equivalents) averaged $14.8 \pm 1.1$ and $4.6 \pm 0.4 \mathrm{nmol} / \mathrm{min}$ per $100 \mathrm{~g}$ body weight, respectively. Pretreatment with phenobarbital significantly increased bile flow, liver weight (from $4.7 \pm 0.1$ to $5.9 \pm 0.3 \mathrm{~g} / 100 \mathrm{~g}$ body weight), and the hepatic concentration of GSH. The increase in the hepatic concentration of GSH was associated with a proportional increase in the biliary concentration of GSH (Fig. 1). As a result of the increased bile flow and the increased concentration, the biliary excretion of GSH demonstrated a disproportionally larger increase than the intrahepatic concentration of GSH but the GSSG/GSH ratio in bile did not change (Table I). The decrease in hepatic GSH produced by the administration of diethyl maleate and acetaminophen was associated with decreased biliary concentrations of GSH. Although consistently lower than the intrahepatic concentration, the biliary concentration of GSH was proportional to the intrahepatic concentration of the tripeptide over a wide range of intrahepatic GSH concentrations (Fig. 1). Separate regression analyses for controls and phenobarbital-treated animals did not reveal any significant differences between the two groups.

In view of this correlation between the hepatic and biliary concentrations of GSH, bile flow should be an additional determinant of the biliary efflux of GSH. Indeed the biliary excretion of GSH in the basal state and during an infusion of BSP-GSH was significantly $(r=0.92)$ correlated with bile flow (Fig. 2). Furthermore, increments or decrements in bile flow resulting from the administration of the tested toxins were associated with proportional changes in the biliary output of GSH

Table I. Effect of Phenobarbital Pretreatment on Biliary Excretion of GSH and GSSG

\begin{tabular}{lcc}
\hline & $\begin{array}{l}\text { Controls } \\
n=21\end{array}$ & $\begin{array}{l}\text { Phenobarbital* } \\
n=7\end{array}$ \\
\hline Bile Flow $(\mu \mathrm{l} / \mathrm{min}$ per $100 \mathrm{~g}$ body weight) & $8.85 \pm 0.22$ & $13.88 \pm 0.78 \S$ \\
GSH (nmol/min per $100 \mathrm{~g}$ body weight) & $14.78 \pm 1.05$ & $33.88 \pm 1.73 \S$ \\
GSSG $(\mathrm{nmol} / \mathrm{min}$ per $100 \mathrm{~g}$ body weight) & $4.59 \pm 0.39$ & $9.75 \pm 0.77 \S$ \\
GSSG/GSH & $0.34 \pm 0.03$ & $0.29 \pm 0.04$ \\
\hline
\end{tabular}

\footnotetext{
* Animals were pretreated with $80 \mathrm{mg} / \mathrm{kg}$ i.p. of phenobarbital for $4 \mathrm{~d}$. The last injection was given $24 \mathrm{~h}$ before determination of the biliary excretion of GSH and GSSG. $\ddagger$ Expressed as GSH equivalents. $\S P<0.01$.
} 


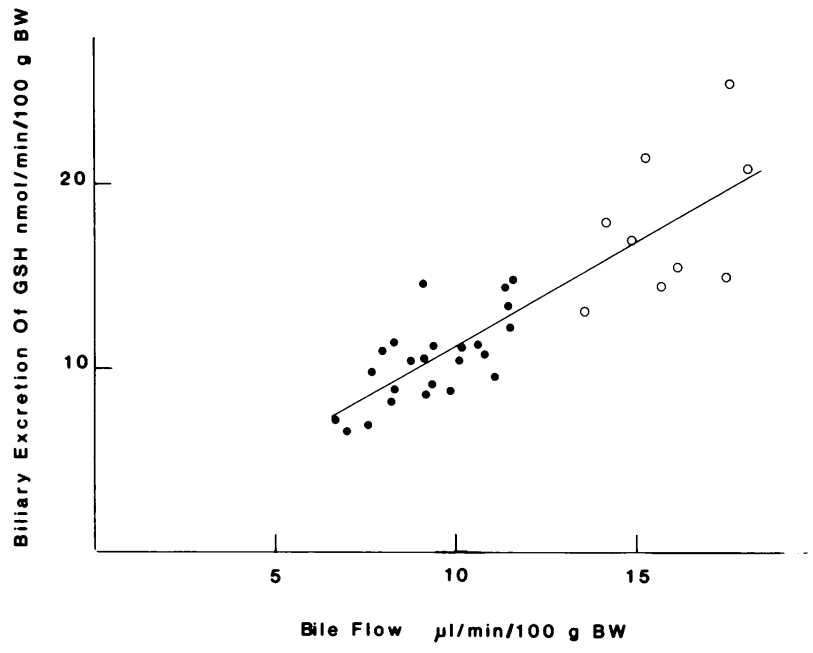

Figure 2. Biliary excretion of GSH as a function of bile flow in control animals $(\bullet)$ and in rats during an infusion of BSP-GSH, 540 $\mathrm{nmol} / \mathrm{min}$ per $100 \mathrm{~g}$ body weight $(\mathrm{BW})(O) . r=0.92$. Separate analysis of the two groups showed identical slopes of the regression.

unless the treatment depleted intrahepatic GSH, as was the case with acetaminophen (data not shown). This observation supports the hypothesis that bile flow is also a major determinant of the biliary excretion of GSH although nonspecific effects of some

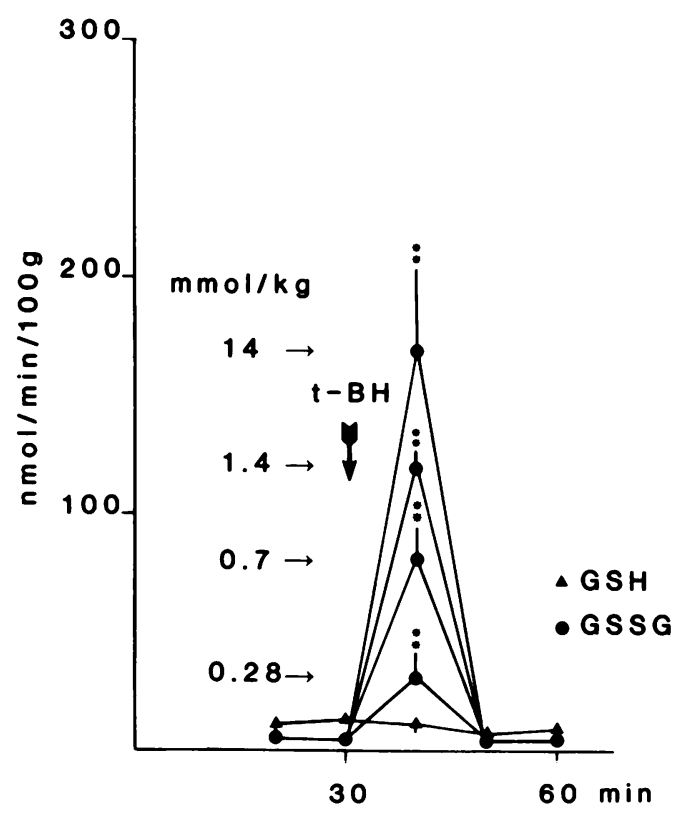

Figure 3. Biliary excretion of GSSG and GSH after the intraperitoneal administration of $0.28-14 \mathrm{mmol} / \mathrm{kg}$ of $t$-butyl hydroperoxide (t-BH) to rats with a biliary fistula. The hydroperoxide resulted in a dose-dependent increase in the biliary excretion of GSSG, whereas the excretion of GSH remained constant (only highest dose data plotted). Mean \pm SE of six rats; GSSG is expressed as GSH equivalents; ${ }^{* *}, P<0.01$. of the toxins on hepatic GSH homeostasis can not be entirely excluded by the present studies. In contrast to GSH, no correlation was found between the biliary excretion of GSSG and changes in bile flow (data not shown).

On the other hand, the biliary excretion of GSSG, but not GSH, was significantly smaller during the infusion of BSP-GSH than in control animals $(2.44 \pm 1.01, n=12$, vs. $4.59 \pm 0.39 \mathrm{nmol}$ GSSG/min per $100 \mathrm{~g}$ body weight, $n=21, P<0.05$ ), suggesting that BSP-GSH might interfere with the biliary excretion of GSSG, which can be considered as the GSH adduct of GSH. Indeed, competition between GSSG and BSP-GSH became more evident following stimulation of the hepatic production of GSSG. The administration of $t$-butyl hydroperoxide led to a dose-dependent increase in the biliary concentration and excretion of GSSG whereas the excretion of GSH remained unchanged (Fig. 3). During the infusion of BSP-GSH at a rate of $540 \mathrm{nmol} / \mathrm{min}$ per $100 \mathrm{~g}$ body weight, the biliary excretion of BSP-GSH reached a plateau at $282 \pm 12 \mathrm{nmol} / \mathrm{min}$ per $100 \mathrm{~g}$. The administration of $1.4 \mathrm{mmol} / \mathrm{kg}$ of $t$-butyl hydroperoxide during this maximal excretion of BSP-GSH led to a significantly smaller increase $(P$ $<0.01$ ) in biliary GSSG (Fig. 4). The rise in biliary GSSG was associated with a comparable fall in the biliary concentration of BSP conjugates (Fig. 4), suggesting a shared transport system. Thin-layer chromatography of bile samples showed that no free BSP was excreted either before or during the peak excretion of GSSG. Since the hepatic concentration of BSP-GSH did not decrease significantly from just before to $10 \mathrm{~min}$ after the ad-

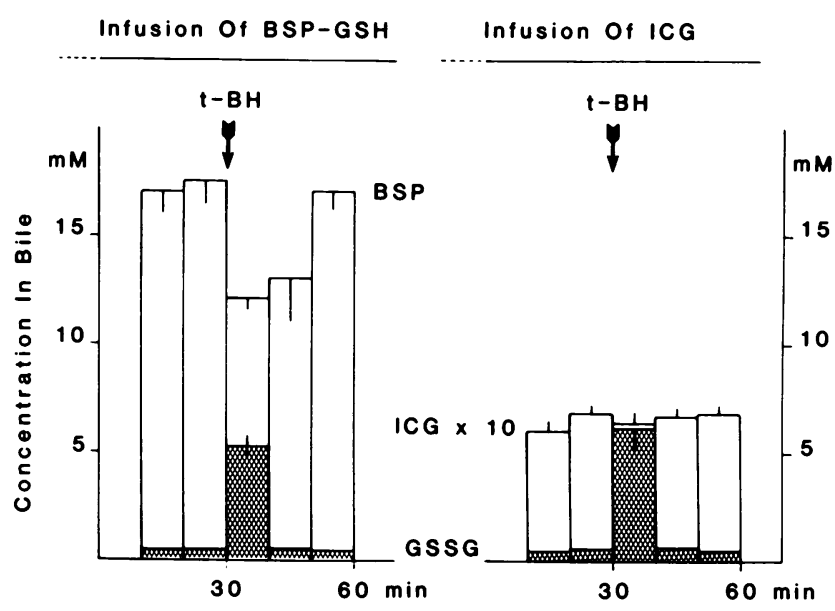

Figure 4. During a constant infusion of BSP-GSH at a rate of 540 $\mathrm{nmol} / \mathrm{min}$ per $100 \mathrm{~g}$ the intraperitoneal administration of $1.4 \mathrm{mmol} /$ $\mathrm{kg}$ of $t$-butyl hydroperoxide ( $\mathrm{t}-\mathrm{BH})$ produced a significantly smaller increase in the biliary concentration of GSSG (hatched bars) than in the control rats shown in Fig. $3(n=7)$. Moreover, the increase in biliary GSSG produced by the hydroperoxide led to a significant decrease in the biliary concentration of BSP-GSH (open bars). In contrast, during a constant infusion $(25 \mathrm{nmol} / \mathrm{min}$ per $100 \mathrm{~g})$ of indocyanine green, which is not conjugated with GSH and is transported by a separate carrier, the maximal biliary concentration of the dye did not change under identical experimental conditions (mean \pm SE, $n=4$ ). 
ministration of $t$-butyl hydroperoxide $(85 \pm 8$ vs. $84 \pm 8 \mu \mathrm{M})$, the time when the biliary excretion of BSP conjugates reached its nadir (Fig. 4), a decreased hepatic uptake of BSP-GSH cannot account for the decrease in its biliary excretion. In contrast to BSP-GSH the biliary concentration of indocyanine green that had reached a plateau indicating maximal biliary excretion of the dye did not decrease as the biliary concentration of GSSG increased after $t$-butyl hydroperoxide (Fig. 4).

On a mole per mole basis the excretion of GSSG was markedly greater after the administration of the redox cycling compounds diquat and nitrofurantoin than after $t$-butyl hydroperoxide (Fig. 5). The administration of diquat and nitrofurantoin during an infusion of BSP-GSH again led to a decrease in the biliary excretion of BSP-conjugates as the excretion of GSSG increased (data not shown). Another redox compound, paraquat, also stimulated biliary GSSG excretion but to a lesser extent than diquat or nitrofurantoin (Table II). Dimethyl sulfoxide, the vehicle for nitrofurantoin, did not affect the basal excretion of GSH or GSSG. Hepatic glutathione reductase activity was not inhibited by the administered dose of nitrofurantoin, averaging $96 \%$ of the activity in control livers.

In vitro, some drugs not known to be hepatotoxins also promote the formation of superoxide anion and $\mathrm{H}_{2} \mathrm{O}_{2}(21)$. We therefore examined the effect of aminopyrine and ethylmorphine on the biliary excretion of GSH and GSSG. Aminopyrine led
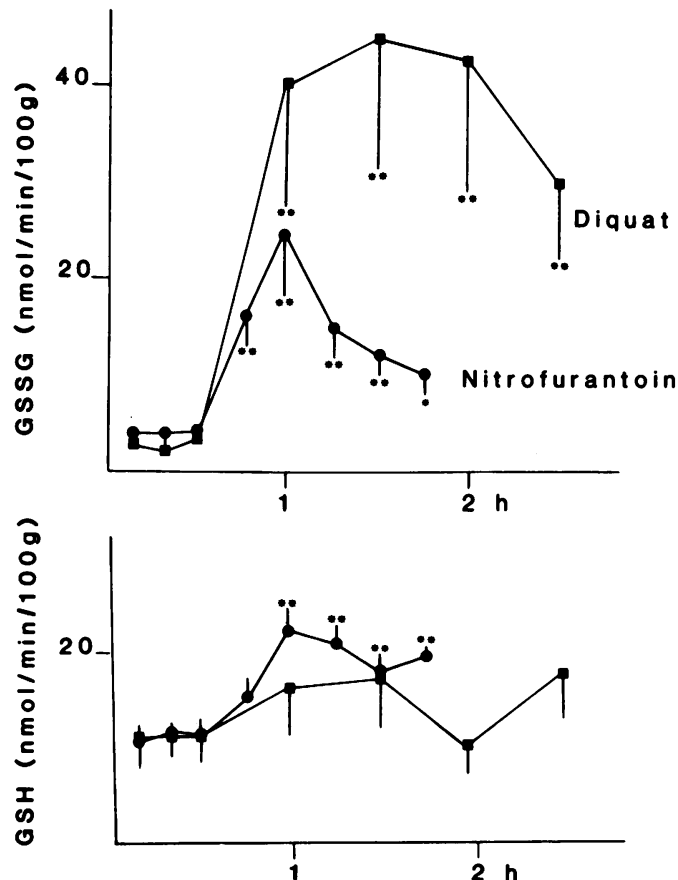

Figure 5. Effect of diquat, $0.09 \mathrm{mmol} / \mathrm{kg}$ intraperitoneally, or nitrofurantoin, $0.2 \mathrm{mmol} / \mathrm{kg}$ i.p., on the biliary excretion of GSH and GSSG. Both compounds stimulate the excretion of GSSG. The excretion of GSH increased slightly and paralleled the fluctuation in bile flow. Mean \pm SE of six rats. GSSG is expressed as GSH equivalents; $*, P<0.05 ;{ }^{* *}, P<0.01$.
Table II. Increment in Biliary Excretion of GSSG After Various Compounds

$\mu$ mol of GSSG*

Diquat $(0.09 \mathrm{mmol} / \mathrm{kg})$

$1.420 \pm 0.058$

Nitrofurantoin $(0.2 \mathrm{mmol} / \mathrm{kg})$

$0.036 \pm 0.007$

Paraquat $(0.3 \mathrm{mmol} / \mathrm{kg})$

$t$-Butyl hydroperoxide $(1.4 \mathrm{mmol} / \mathrm{kg})$

$0.012 \pm 0.001$

Aminopyrine $(0.8 \mathrm{mmol} / \mathrm{kg})$

Ethylmorphine $(0.08 \mathrm{mmol} / \mathrm{kg})$

Iproniazid $(0.4 \mathrm{mmol} / \mathrm{kg})$

Carbon tetrachloride $(10 \mathrm{mmol} / \mathrm{kg})$

Dimethylnitrosamine $(0.4 \mathrm{mmol} / \mathrm{kg})$

Furosemide $(1.2 \mathrm{mmol} / \mathrm{kg})$

Chloroform $(12 \mathrm{mmol} / \mathrm{kg})$

Acetaminophen $(6.6 \mathrm{mmol} / \mathrm{kg})$

$0.004 \pm 0.0004$

$0.004 \pm 0.001$

no increment in GSSG

no increment in GSSG

no increment in GSSG

no increment in GSSG no increment in GSSG decrement in GSSG decrement in GSSG

* Expressed as GSH equivalents, excreted during each experiment per micromole of the administered compound.

to a small, dose-dependent increase in biliary GSSG (Fig. 6) but in contrast to the redox-cycling compounds this occurred only in association with a proportional increase in biliary GSH such that the ratio of GSSG to GSH did not change (Fig. 7).

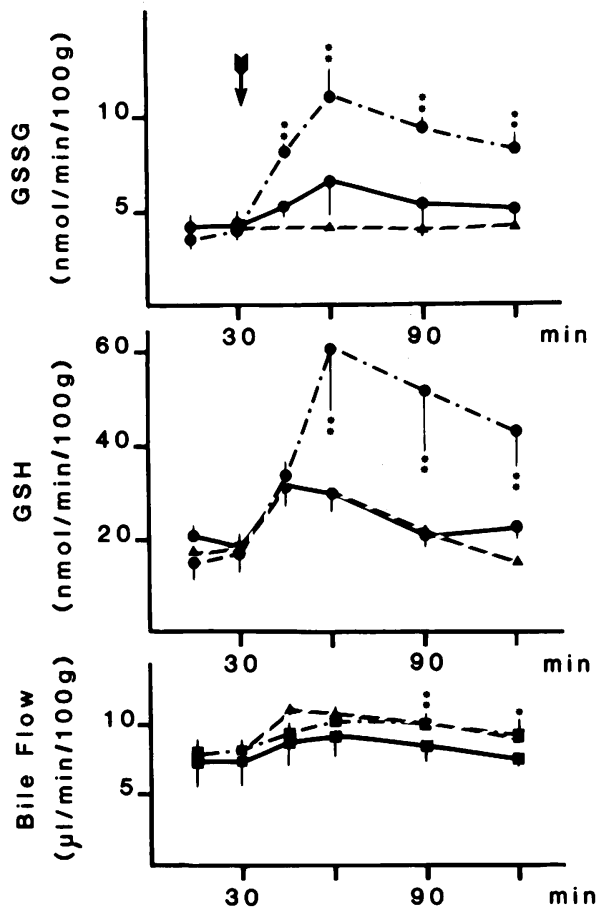

Figure 6. Effect of aminopyrine (๑), $80 \mu \mathrm{mol} / 100 \mathrm{~g}$ i.p. (dotted line) and $8 \mu \mathrm{mol} / 100 \mathrm{~g}$ i.p. (solid line) and $8 \mu \mathrm{mol} / 100 \mathrm{~g}$ i.p. ( $\Delta$ ) of ethylmorphine on the biliary excretion of GSH. Aminopyrine increased the biliary excretion of GSSG slightly but the GSSG/GSH ratio did not change because of the proportional increase in the efflux of GSH. Mean \pm SE of five rats. GSSG is expressed as GSH equivalents; ${ }^{* *}, P<0.01 ;{ }^{*}, P<0.05$. 


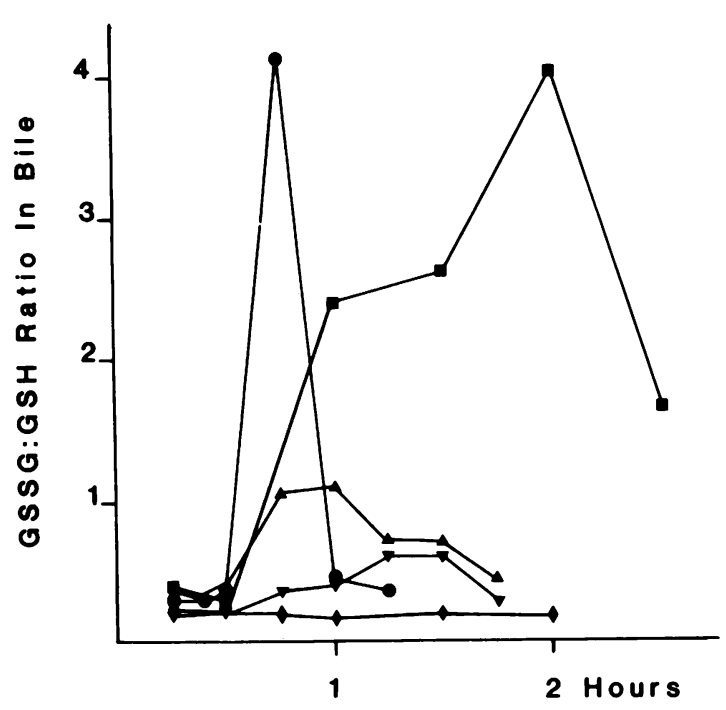

Figure 7. Ratio of GSSG/GSH in bile for the five chemicals that increased biliary GSSG, diquat $(\boldsymbol{\nabla})$, nitrofurantoin $(\boldsymbol{\Delta})$, paraquat $(\boldsymbol{\nabla})$, $t$-butyl hydroperoxide $(1.4 \mathrm{mmol} / \mathrm{kg}$ dose $)(\bullet)$, and aminopyrine $(0.8$ $\mathrm{mmol} / \mathrm{kg}$ dose) $(\diamond)$. Average of three to eight animals. All compounds producing an oxidant stress significantly increase the GSSG/GSH ratio but the ratio does not change after aminopyrine in spite of a significant increase in the biliary excretion of GSSG.

Ethylmorphine did not affect biliary efflux of GSSG at a dose of $8 \mu \mathrm{mol} / 100 \mathrm{~g}$ body weight (Fig. 6).

Acetaminophen, an analgesic that is metabolized to an electrophilic metabolite which depletes GSH, has also been postulated to produce an oxidative stress (22). We therefore tested acetaminophen and another compound, chloroform, the electrophilic phosgene metabolite of which also moderately depletes GSH. Acetaminophen resulted in a marked choleresis associated with an early increase in the biliary excretion of GSH (Fig. 8). At the time of the peak biliary excretion of GSH the biliary concentration of GSH was not significantly higher than in the basal state, indicating that the increment in bile flow was responsible for the increment in GSH excretion. At later time points, however, the biliary concentration and the biliary excretion of GSH and GSSG decreased, reflecting the depletion of intracellular GSH. In control animals studied for the same period of time, the biliary concentrations of GSSG and GSH remained constant $(102 \pm 12 \%$ of the concentration in the first two bile samples at $4 \mathrm{~h}[n=5])$ and their biliary excretion tended to decrease, albeit not statistically significant, in parallel to the decrease in bile flow that is seen over this period of time without replacement of bile acids lost via the cannula. Chloroform did not stimulate bile flow and thus did not increase the biliary excretion of GSH. Like acetaminophen, chloroform tended to decrease GSH at later time points. In contrast, no decrease in biliary GSH or GSSG was observed after the administration of two other compounds, furosemide and dimethylnitrosamine, which generate electrophilic metabolites but do not deplete hepatic GSH $(5,23,24)$. As was the case with
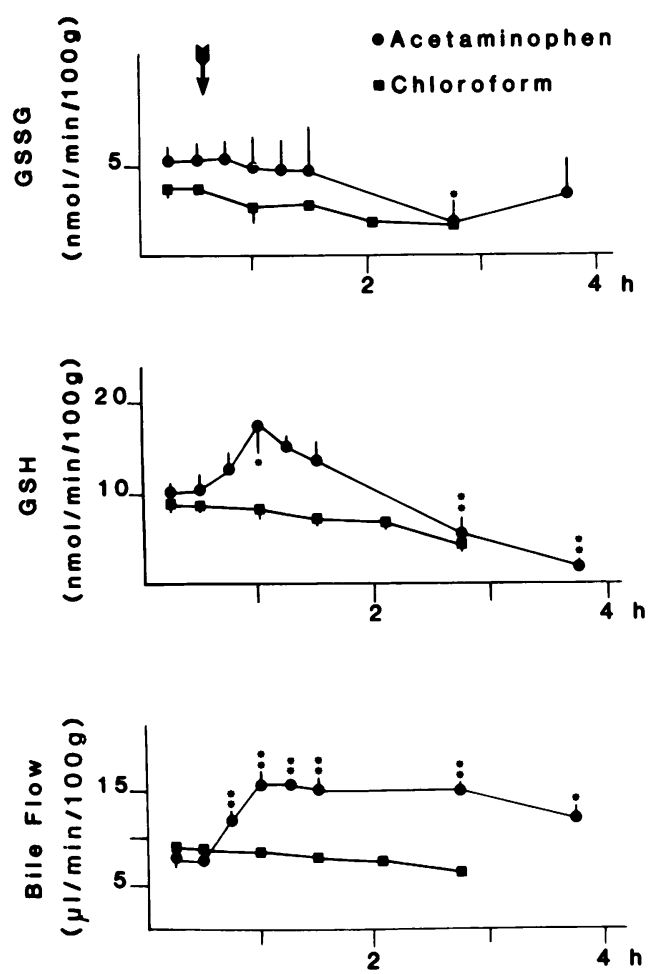

Figure 8. Biliary excretion of GSH and GSSG after the administration of $6.6 \mathrm{mmol} / \mathrm{kg}$ i.p. of acetaminophen and $12 \mathrm{mmol} /$ $\mathrm{kg}$ i.p. of chloroform. After an initial increase in the biliary excretion of GSH associated with a marked increase in bile flow, acetaminophen led to a progressive decrease in biliary GSH, reflecting the intracellular depletion of GSH. Neither acetaminophen nor chloroform stimulated the excretion of GSSG. Mean \pm SE of four rats. GSSG expressed as GSH equivalents. ${ }^{* *}, P<0.01$; ${ }^{*}, P<0.05$.

acetaminophen and aminopyrine, furosemide stimulated bile flow and resulted in an early rise in the efflux of GSH but without altering the biliary concentration of GSH (Fig. 9). Thus, none of the four compounds yielding electrophilic metabolites significantly increased the excretion of GSSG or the GSSG/ GSH ratio in bile.

An additional class of hepatotoxins that might produce oxidative stress is a chemical that yields organic radical metabolites that can react with membrane lipids to abstract hydrogen atoms, thereby leading to the formation of lipid hydroperoxides in the presence of oxygen (25). The latter should stimulate the generation of GSSG if they are substrates for glutathione peroxidases, as has been postulated $(9,25)$. However, carbon tetrachloride did not increase the biliary excretion of GSSG (Fig. 10) although similar doses have been shown to produce lipid peroxidation in vivo in the same animal model $(24,25)$. Similarly, iproniazid, another compound activated to an alkylating radical (26), also failed to stimulate the excretion of GSH and GSSG (Fig. 10).

\section{Discussion}

The characteristics of the biliary excretion of GSH and GSSG are quite distinct. The intrahepatic concentration of GSH and 

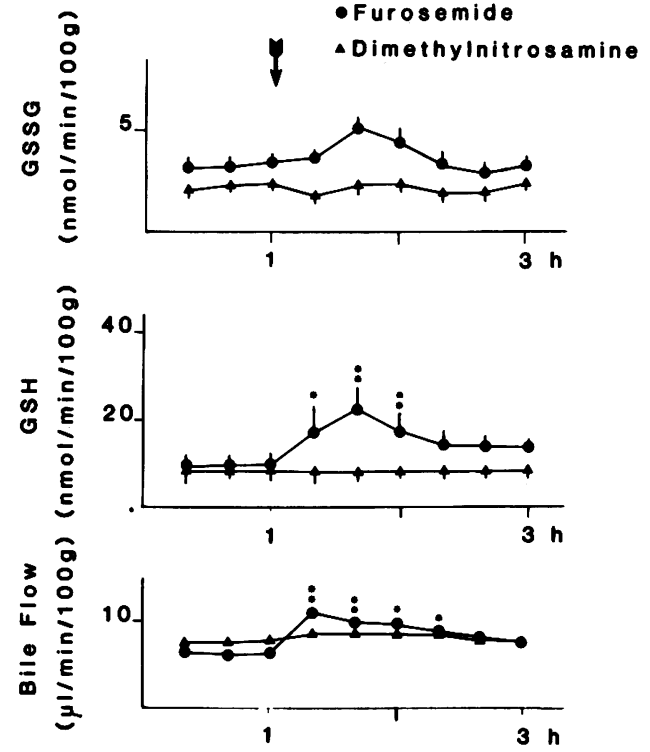

Figure 9. Biliary excretion of GSSG and GSH after the administration of furosemide $(n=5)$ and dimethylnitrosamine $(n$ $=3$ ), two compounds generating electrophilic metabolites that do not deplete hepatic GSH. Mean \pm SE. GSSG is expressed as GSH equivalents. ${ }^{* *} P<0.01{ }^{*} P<0.05$.

the rate of bile flow appear to be the major determinants of biliary efflux of GSH. Because of the activity of hepatic gammaglutamyl transferase, which is responsible for the initial step in the degradation of GSH and GSSG and is mainly located in the epithelial cells of the bile ducts (27-30), the actual amount of GSH leaving the hepatocytes is probably underestimated. Even in the rat, which has a much lower hepatic activity of gamma-glutamyl transferase compared with guinea pig and man
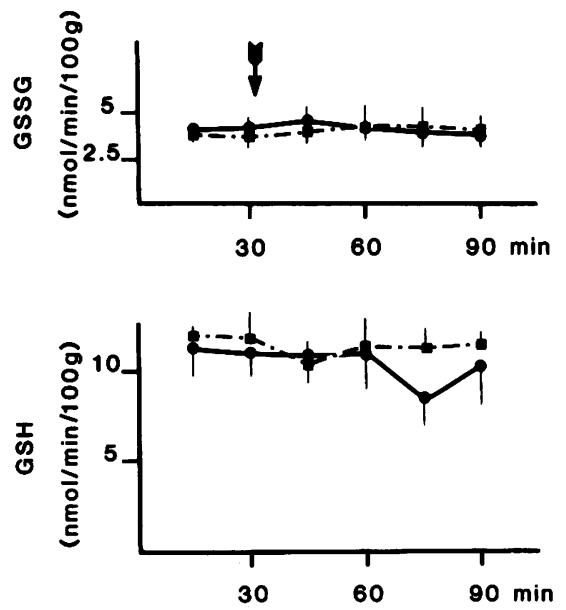

Figure 10. Absence of an effect of carbon tetrachloride ( $(0), 10$ $\mathrm{mmol} / \mathrm{kg}$ i.p., and of iproniazid (घ), $0.4 \mathrm{mmol} / \mathrm{kg}$ i.p., on the biliary excretion of GSH and GSSG. Mean \pm SE of eight animals. GSSG is expressed as GSH equivalents.
(31), substantial quantities of cysteinylglycine appear in bile (B. Lauterburg, unpublished observation) but it is not known how much of the biliary cysteinylglycine originates from glutathione. Thus, the activity of gamma-glutamyl transferase should be considered when comparing the amount of biliary GSH excreted in different species.

The observation that the biliary concentration of GSH consistently amounts to $\sim 30 \%$ of the intrahepatic concentration over a wide range of hepatic GSH levels is compatible with the view that intracellular GSH passes into bile passively along a concentration gradient. That the efflux of GSH is a passive phenomenon is further supported by the fact that in contrast to GSSG, GSH is not preferentially excreted into bile (14). The amount of GSH appearing in bile accounts for only $\sim 25 \%$ of the estimated in vivo rate of hepatic synthesis of $55 \mathrm{nmol} / \mathrm{min}$ per $100 \mathrm{~g}$ body weight (32). Most of the remaining hepatic production of GSH is accounted for by efflux on the sinusoidal side into blood $(12,13)$. Experiments with perfused rat liver demonstrate a preferential flow and temperature-dependent (33) efflux of GSH into the perfusate and are consistent with our in vivo data.

In contrast to GSH, on the other hand, GSSG is almost exclusively excreted into bile in the perfused rat liver (14). Moreover, the biliary concentration of GSSG is $\sim 20$-fold higher than the intrahepatic concentration of the disulfide (34), indicating the presence of a concentrating mechanism. Unfortunately, the concentration of GSSG in bile canaliculi is not known. Since GSH undergoes rapid oxidation in bile $(15,35)$ an artifactual increase in biliary GSSG due to oxidation of GSH during its passage through the biliary tree cannot be ruled out. In addition, the biliary GSH/GSSG ratio may be modulated by gamma-glutamyltransferase. In vitro GSH is a much better substrate for the enzyme than GSSG (30) such that the enzyme could lead to an artifactual decrease in the biliary concentration of GSH relative to GSSG. Inhibition of the enzyme by any of the toxins or their metabolites might thus be expected to decrease the GSSG/GSH ratio in bile and possibly mask an oxidant stress. However, the excretion of GSH would then increase because of the decreased extrahepatic catabolism (35). Conversely, activation of the enzyme might result in an increase in the GSSG/GSH ratio associated with a decreased excretion of GSH and GSSG.

Nevertheless, even though extrahepatic oxidation and metabolism might be responsible for some of the GSSG excreted in the basal state, such mechanisms cannot account for the marked increase in biliary GSSG observed after the administration of $t$-butyl hydroperoxide, diquat, or nitrofurantoin. Addition of these compounds to bile in vitro does not enhance the oxidation of GSH. The striking increase in the GSSG/GSH ratio occurring concomitantly with a marked increase in the excretion of GSH plus GSSG therefore indicates that the GSSG is generated intracellularly and not extrahepatically under these circumstances. As demonstrated in the perfused rat liver, the intrahepatic concentration of GSSG indeed increases markedly under these conditions of oxidative stress but is still $\sim 50$ times lower than the biliary concentration (34), clearly indicating the 
presence of a concentrating transport mechanism of GSSG. The existence of such a transport mechanism is further supported by the observation that GSSG appears to share its excretory mechanism with other GSH adducts. The rise in biliary GSSG after $t$-butyl hydroperoxide was associated with a comparable fall in the biliary concentration and the excretory maximum for BSP conjugates. Since the hepatic concentration of BSPGSH did not decrease during the peak excretion of GSSG, the decreased biliary excretion of BSP conjugates cannot be accounted for by a decreased hepatic uptake of BSP-GSH. Moreover, since BSP-GSH was the infused compound, competition for GSH for conjugation with free BSP vs. formation of GSSG cannot explain the decreased excretion of BSP conjugates. This is reflected by the lack of any effect of BSP-GSH on the biliary excretion of GSH itself. The inhibition of the biliary excretion of the GSH adduct of 1-chloro-2,4-dinitrobenzene by GSSG, and vice versa, which has been demonstrated in the perfused rat liver, provide further evidence for a shared biliary excretory mechanism for glutathione adducts including GSSG itself (36). Of course, unknown or nonspecific effects of $t$-butyl hydroperoxide on anion transport cannot be totally excluded. Nevertheless, the peroxide had no effect on an anion (indocyanine green) transported into bile by a carrier separate from the carrier for BSP-GSH and GSSG.

Nitrofurantoin and the bipyridylium compounds diquat and paraquat are thought to produce superoxide anion during NADPH-dependent redox cycling $(4,37,38)$. In the process of reducing superoxide, several intermediates including $\mathrm{H}_{2} \mathrm{O}_{2}$ are formed that are eventually metabolized to water (39). Reduction of the $\mathrm{H}_{2} \mathrm{O}_{2}$ intermediate by glutathione peroxidases is the major detoxifying pathway outside the peroxisomal compartment $(9$, 40). Because of redox cycling, one mole of one of these compounds should generate many moles of $\mathrm{H}_{2} \mathrm{O}_{2}$ and hence many moles of GSSG via glutathione peroxidase. Indeed, the administration of diquat and nitrofurantoin led to a marked increase in the biliary excretion of GSSG that greatly exceeded on a mole per mole basis the generation of GSSG by direct substrates for glutathione peroxidase such as $t$-butyl hydroperoxide (Table II). Inhibition of hepatic glutathione reductase might in part be responsible for the increased biliary excretion of GSSG caused by some of the compounds. Nitrofurantoin, for instance, has been shown to inhibit glutathione reductase in vitro (41). However, the compound does not inhibit the reductase if administered in vivo $(41,42)$, as also confirmed by our studies, indicating that the increased biliary excretion of GSSG does not result from a decreased reduction of intrahepatic GSSG.

After the administration of paraquat, the biliary excretion of GSSG increased only moderately although it still exceeded the generation of GSSG by $t$-butyl hydroperoxide (Table II). The moderate effect of paraquat relative to diquat on biliary GSSG in vivo, which stands in contrast to the greater pulmonary toxicity of paraquat, is probably due to the greater accumulation of paraquat in the lungs (43), since paraquat markedly increases the intrahepatic concentration of both GSSG and mixed disulfides (44) as well as the biliary excretion of GSSG (45) when injected directly in the isolated perfused rat liver.
Some drugs such as aminopyrine and ethylmorphine can increase the formation of $\mathrm{H}_{2} \mathrm{O}_{2}$ by hepatic microsomal preparations apparently by the release of superoxide anion from monoreduced oxycytochrome P450 (21). From experiments in the perfused rat liver, however, other investigators have proposed that any increases in GSSG produced by aminopyrine in phenobarbital-induced animals do not result from an increased formation of $\mathrm{H}_{2} \mathrm{O}_{2}$ intracellularly. Rather, they postulate that competition for the available NADPH, which also serves as the hydrogen donor for the metabolism of aminopyrine by P450 monooxygenases, limits the availability of NADPH for reduction of GSSG $(40,46)$.

The conclusion from these in vitro studies that aminopyrine does not produce a significant oxidative stress is consistent with the present results in noninduced animals in vivo. The increase in biliary GSSG after aminopyrine observed in our experiments was associated with a marked increase in GSH but no change in the GSSG/GSH ratio (Fig. 7). This parallel increase in GSH and GSSG suggests that the increased excretion of GSSG resulted from extrahepatic oxidation of biliary GSH rather than from increased intrahepatic generation of GSSG. Whenever the biliary excretion of GSH increased markedly in experiments with other compounds, biliary GSSG also increased (e.g., Table I, Fig. 9). As discussed previously, inhibition of gamma glutamyl-transferase could explain the increase in biliary GSH and GSSG after aminopyrine (35). However, aminopyrine stimulates rather than inhibits the enzyme (47). The lack of stimulation of GSSG excretion by ethylmorphine, which is even more potent than aminopyrine in producing $\mathrm{H}_{2} \mathrm{O}_{2}$ in the $\mathrm{P} 450$ system in vitro, also supports the view that minimal oxidative stress is produced by such compounds in noninduced rats in vivo. Small increases in biliary GSSG must therefore be interpreted with caution and probably should not be considered to reflect an oxidative stress unless they are associated with a significant increase in the GSSG/ GSH ratio.

No evidence of oxidative stress was seen with acetaminophen and chloroform, chemicals that yield electrophilic metabolites that selectively deplete hepatic GSH and lead to a decrease in the biliary excretion of GSSG and GSH. The increased biliary excretion of GSH that occurred shortly after the administration of acetaminophen was most likely because of the marked increase in bile flow (Fig. 8). The choleresis-associated increase in efflux, however, was soon offset by the progressive depletion of intrahepatic GSH. During the period of time we followed the biliary excretion of GSH and GSSG, significant depletion of hepatic GSH and covalent binding occurs in the same animal model (48). The absence of a concomitant increase in biliary GSSG does not support the hypothesis of Wendel and Feuerstein (22) that oxygen radical-induced lipid peroxidation contributes significantly to the toxicity of acetaminophen. Chemicals that yield electrophilic metabolites that do not preferentially combine with and thereby deplete hepatic GSH but cause extensive hepatic necrosis, such as dimethylnitrosamine $(5,49)$, also did not alter the biliary excretion of GSSG (Fig. 9).

In addition to $\mathrm{H}_{2} \mathrm{O}_{2}$, lipid hydroperoxides generated by organic radical attack on membrane polyunsaturated fatty acids 
have been considered to be substrates for glutathione peroxidases $(50,51)$. It is therefore surprising that a toxic dose of carbon tetrachloride did not affect the biliary excretion of GSSG, since an identical dose leads to peroxidation of lipids as demonstrated by a marked increase in the exhalation of ethane and pentane and the formation of hydroxy fatty acids in membrane lipids (24). In a preliminary experiment mentioned elsewhere (52) and that is included in the data presented in Fig. 10, carbon tetrachloride was thought to produce a moderate increase in biliary GSSG. However, subsequent analysis of this study showed that the GSSG/GSH ratio in bile did not increase significantly, owing to a proportional increase in biliary GSH. Similarly, iproniazid, which also is metabolized to an organic radical, did not increase the biliary excretion of GSSG. Accordingly, the lack of change in biliary excretion of GSSG at the time of ongoing lipid peroxidation indicates that membrane-bound hydroperoxides may not be good substrates for glutathione peroxidases (53). This interpretation is further substantiated by the observation that depletion of hepatic GSH by administration of diethyl maleate does not increase the formation of ethane and pentane or of hepatic hydroxy fatty acids after carbon tetrachloride (24).

In conclusion, our data indicate that efflux of GSH from the liver occurs passively along a concentration gradient such that the intrahepatic concentration of GSH and bile flow are the major determinants of biliary excretion of GSH. In contrast, GSSG appears to be actively secreted into bile against a steep concentration gradient. The GSSG shares this transport mechanism with other GSH adducts. Our studies with selected model compounds demonstrate that an increased biliary excretion of GSSG associated with an increase in the GSSG/GSH ratio in bile is a sensitive index of oxidative stress in vivo. The quantitative analysis of biliary output of GSSG can identify compounds that undergo redox cycling, thereby producing more GSSG on a molar basis than a reference hydroperoxide. Because the GSH and GSSG excreted into bile cannot be recovered by the liver (54), the increased demand on the GSH pool resulting from a prolonged oxidative stress could eventually deplete intracellular GSH (44). Compounds leading to lipid peroxidation via alkylating radicals, on the other hand, do not increase the generation of GSSG, probably because membrane-bound lipid hydroperoxides are not accessible to glutathione peroxidases. The biliary excretion of GSSG thus complements other in vivo parameters for the study of reactive intermediates of xenobiotics such as the determination of covalent binding, the formation of lipid hydroxy acids, and the depletion of intracellular GSH (5).

\section{Acknowledgments}

Dr. Mitchell is a Burroughs Wellcome Scholar in Clinical Pharmacology.

This work was supported by grants GM 26611 and GM 13901 of the National Institute for General Medical Sciences.

\section{References}

1. Mitchell, J. R., and D. J. Jollow. 1975. Metabolic activation of drugs to toxic substances. Gastroenterology. 68:392-410.
2. Mitchell, J. R., R. J. McMurtry, C. N. Statham, and S. D. Nelson. 1977. Molecular basis for several drug-induced nephropathies. Am. J. Med. 62:518-526.

3. Boyd, M. R. 1980. Biochemical mechanisms in chemical-induced lung injury: roles of metabolic activation. CRC Crit. Rev. Toxicol. 7:103176.

4. Bus, J. S., and J. E. Gibson. 1967. Paraquat toxicity: proposed mechanisms of toxicity involving lipid peroxidation. Environ. Health Perspect. 16:139-146.

5. Mitchell, J. R., H. Hughes, B. H. Lauterburg, and C. V. Smith. 1982. Chemical nature of reactive intermediates as determinants of toxicologic responses. Drug Metab. Rev. 13:539-553.

6. Fisher, H. K., J. A. Clements, and R. R. Wright. 1973. Enhancement of oxygen toxicity by the herbicide paraquat. Am. Rev. Resp. Dis. 107:246-252.

7. Boyd, M. R., G. L. Catignani, H. A. Sasame, J. R. Mitchell, and A. W. Stiko. 1979. Acute pulmonary injury in rats by nitrofurantoin and modification by vitamin E, dietary fat and oxygen. Am. Rev. Resp. Dis. 120:93-99.

8. Frank L. 1981. Prolonged survival after paraquat. Role of the lung antioxidant enzyme systems. Biochem. Pharmacol. 70:2319-2324.

9. Flohé, L. 1979. Glutathione peroxidase: fact and fiction. In Oxygen-free radicals and tissue damage. Ciba Foundation Symposium 65. Excerpta Medica, Amsterdam. 95-113.

10. Chance, B., H. Sies, and A. Boveris. 1979. Hydroperoxide metabolism in mammalian organs. Physiol. Rev. 59:527-605.

11. Sies, H., and K. H. Summer. 1975. Hydroperoxide metabolizing systems in rat liver. Eur. J. Biochem. 57:503-512.

12. Anderson, M. E., R. J. Bridges, and A. Meister. 1980. Direct evidence for inter-organ transport of glutathione and that the nonfiltration renal mechanism for glutathione utilization involves gamma-glutamyl transpeptidase. Biochem. Biophys. Res. Commun. 96:848-853.

13. Lauterburg, B. H., J. D. Adams, and J. R. Mitchell. 1983. Efflux of glutathione from the liver accounts for total hepatic production in vivo. Fed. Proc. 42:890.

14. Sies, H., A. Wahlländer, and C. H. Waydhas. 1978. Properties of glutathione disulfide (GSSG) and glutathione-S-conjugate release from perfused rat liver. In Functions of Glutathione in Liver and Kidney. $\mathrm{H}$. Sies and A. Wendel, editors. Springer Publishing Co., Inc., New York. 120-126.

15. Eberle, D., R. Clarke, and N. Kaplowitz. 1981. Rapid oxidation in vitro of endogenous and exogenous glutathione in bile of rats. J. Biol. Chem. 256:2115-2117.

16. Whelan, G., J. Hoch, and B. Combes. 1970. A direct assessment of the importance of conjugation of biliary transport of sulfobromophthalein sodium. J. Lab. Clin. Med. 75:542-557.

17. Horak, W., G. Grabner, and G. Paumgartner. 1973. Inhibition of bile salt-independent bile formation by indocyanine green. Gastroenterology. 64:1005-1012.

18. Tietze, F. 1969. Enzymic method for quantitative determination of nanogram amounts of total and oxidized glutathione. Anal. Biochem. 27:502-522.

19. Griffith, O. W. 1980. Determination of glutathione and glutathione disulfide using glutathione reductase and 2-vinylpyridine. Anal. Biochem. 106:207-212.

20. Pinto, R. E., and W. Bartley. 1969. The effect of age and sex on glutathione reductase and glutathione peroxidase activities and on aerobic glutathione oxidation in rat liver homogenates. Biochem. $J$. 112:109-115.

21. Gillette, J. R., B. B. Brodie, and B. N. La Du. 1957. The oxidation 
of drugs by liver microsomes: on the role of TPNH and oxygen. $J$. Pharmacol. Exp. Ther. 119:532-540.

22. Wendel, A., and S. Feuerstein. 1981. Drug-induced lipid peroxidation in mice. I. Modulation by monooxygenase activity, glutathione and selenium status. Biochem. Pharmacol. 30:2513-2520.

23. Mitchell, J. R., W. Z. Potter, J. A. Hinson, and D. J. Jollow. 1974. Hepatic necrosis caused by furosemide. Nature (Lond.). 251:508511.

24. Smith, C. V., H. Hughes, B. H. Lauterburg, and J. R. Mitchell. The chemical nature of reactive metabolites determines their biological interactions with glutathione. In the Fifth Karolinska Institute Nobel Conference. Functions of Glutathione-Biochemical, Physiological and Toxicological Aspects. A. Larsson, B. Mannervik, and S. Orrenius, editors. Raven Press, New York. In press.

25. Recknagel, R. O., E. A. Glende, and A. M. Hruszkewycz. 1977. Chemical mechanisms in carbon tetrachloride toxicity. In Free Radicals in Biology. W. A. Pryor, editor. Academic Press, Inc., New York. III:97132.

26. Nelson, S. D., J. R. Mitchell, W. R. Snodgrass, and J. A. Timbrell. 1978. Hepatotoxicity and metabolism of iproniazid and isopropylhydrazine. J. Pharmacol. Exp. Ther. 206:574-585.

27. Albert, Z., M. Orlowski, and A. Szewczuk. 1961. Histochemical demonstration of gamma-glutamyl transpeptidase. Nature (Lond.). 191:767-768.

28. Glenner, G. G., J. E. Folk, and P. J. McMillan. 1962. Histochemical demonstration of gamma-glutamyl transpeptidase-like activity. J. Histochem. Cytochem. 10:481-489.

29. Rutenberg, A. M., H. Kim, J. W. Fischbein, J. S. Hanker, H. L. Wasserkrug, and A. M. Seligman. 1969. Histochemical and ultrastructural demonstration of gamma-glutamyl transpeptidase activity. $J$. Histochem. Cytochem. 17:517-526.

30. Tate, S. S., and A. Meister. 1974. Interaction of gamma-glutamyl transpeptidase with amino acids, dipeptides, and derivatives and analogs of glutathione. J. Biol. Chem. 249:7593-7602.

31. Albert, Z., J. Orlowska, M. Orlowski, and A. Szewczuk. 1964 Histochemical and biochemical investigations of gamma-glutamyl transpeptidase in the tissue of man and laboratory rodents. Acta Histochem. 18:78-89.

32. Lauterburg, B. H., and J. R. Mitchell. 1981. Regulation of hepatic glutathione turnover in rats in vivo and evidence for kinetic homogeneity of the hepatic glutathione pool. J. Clin. Invest. 67:1415-1424.

33. Bartoli, G. M., and H. Sies. 1978. Reduced and oxidized glutathione efflux from liver. FEBS (Fed. Eur. Biochem. Soc.) Lett. 86:8991.

34. Akerboom, T. P. M., M. Bilzer, and H. Sies. 1982. The relationship of biliary glutathione disulfide efflux and intracellular glutathione disulfide content in perfused rat liver. J. Biol. Chem. 257:4248-4252.

35. Abbott, W. A., and Meister, A. 1982. Biliary glutathione; hepatic and pancreatic contributions. Fed. Proc. 41:1430.

36. Akerboom, T. P. M., M. Bilzer, and H. Sies. 1982. Competition between transport of glutathione disulfide (GSSG) and glutathione Sconjugates from perfused rat liver into bile. FEBS (Fed. Eur. Biochem. Soc.) Lett. 140:73-76.

37. Mason, R. P., and J. L. Holtzman. 1975. Role of catalytic superoxide formation in the oxygen inhibition of nitroreductase. Biochem. Biophys. Res. Commun. 67:1267-1274.

38. Sasame, H. A., and M. R. Boyd. 1979. Superoxide and hydrogen peroxide production and NADPH oxidation stimulated by nitrofurantoin in lung microsomes: possible implications for toxicity. Life Sci. 24:10911096

39. Kappus, H., and H. Sies. 1981. Toxic drug effects associated with oxygen metabolism: redox cycling and lipid peroxidation. Experientia (Basel). 37:1233-1241.

40. Oshino, N., and B. Chance. 1977. Properties of glutathione release observed during reduction of organic hydroperoxide, demethylation of aminopyrine and oxidation of some substances in perfused rat liver and their implications for the physiological function of catalase. Biochem. J. 162:509-525.

41. Buzard, J. A., F. Kopko, and M. F. Paul. 1960. Inhibition of glutathione reductase by nitrofurantoin. J. Lab. Clin. Med. 56:884-890.

42. Dunbar, J. R., A. J. DeLucia, and L. R. Bryant. 1981. Effects of nitrofurantoin on the glutathione redox status and related enzymes in the isolated, perfused rabbit lung. Res. Commun. Chem. Pathol. Pharmacol. 34:485-492.

43. Rose, M. S., L. L. Smith, and I. Wyatt. 1974. Evidence for energy-dependent accumulation of paraquat into rat lung. Nature (Lond.). 252:314-315.

44. Brigelius, R., R. Lenzen, and H. Sies. 1982. Increase in hepatic mixed disulphide and glutathione disulphide levels elicited by paraquat. Biochem. Pharmacol. 31:1637-1641.

45. Brigelius, R., and M. S. Anwer. 1981. Increased biliary GSSGsecretion and loss of hepatic glutathione in isolated perfused rat liver after paraquat treatment. Res. Commun. Chem. Pathol. Pharmacol. 31: 493-502.

46. Sies, H., G. M. Bartoli, R. F. Burk, and C. H. Waydhas. 1978. Glutathione efflux from perfused rat liver after phenobarbital treatment during drug oxidation, and in selenium deficiency. Eur. J. Biochem. 89:113-118.

47. Igarashi, T., T. Satoh, K. Hoshi, K. Ueno, and H. Kitagawa. 1982. Perturbation of hepatic glutathione level and glutathione-related enzyme activities by repeated administration of aminopyrine in rats. Life Sci. 31:2655-2665.

48. Lauterburg, B. H., and J. R. Mitchell. 1982. Toxic doses of acetaminophen suppress hepatic glutathione synthesis in rats. Hepatology. $2: 8-12$.

49. Skaare, J. U., and I. Nafstad. 1978. Interaction of vitamin E and selenium with the hepatotoxic agent dimethylnitrosamine. Acta Pharmacol. Toxicol. 43:119-128.

50. Flohé, L., and R. Zimmermann. 1970. The role of GSH peroxidase in protecting the membrane of rat liver mitochondria. Biochim. Biophys. Acta. 223:210-213.

51. Christophersen, B. O. 1969. Reduction of linolenic acid hydroperoxide by a glutathione peroxidase. Biochim. Biophys. Acta. 176:463470.

52. Mitchell, J. R., G. B. Corcoran, C. V. Smith, H. Hughes, and B. H. Lauterburg. 1981. Alkylation and peroxidation injury from chemically reactive metabolites. In Biological Reactive Intermediates. R. Snyder, D. Parke, J. Kocsis, and D. Jollow, editors. Plenum Press, New York. 2:199-224.

53. McCay, P. B., D. D. Gibson, K. L. Fond, and K. R. Hornbrook. 1976. Effect of glutathione peroxidase activity on lipid peroxidation in biological membranes. Biochem. Biophys. Acta. 431:459-468.

54. Hahn, R., and W. Oberrauch. 1978. Unidirectional transport of reduced glutathione in rat liver and its metabolization in the extracellular space. In Functions of glutathione in liver and kidney. H. Sies and A. Wendel, editors. Springer Publishing Co., Inc., New York, 3240. 